\title{
Publication channels
}

Richard Walker, Pascal Rocha da Silva

\section{Source}

Richard Walker, Pascal Rocha da Silva. (2015). Emerging trends in peer review-a survey.

Front. Neurosci., vol. 9.

Publication channels are media (e.g., preprint-servers, print or online journals) that allow the publication of papers, reviews of papers or user commentary on papers. 